\title{
OPTICAL MASS ESTIMATES OF GALAXY CLUSTERS
}

\author{
Marisa Girardi ${ }^{1,2,3}$, Giuliano Giuricin ${ }^{2,3}$, Fabio Mardirossian ${ }^{1,2,3}$, Marino Mezzetti ${ }^{2,3}$, \\ AND WALTER BOSCHIN ${ }^{2,3}$ \\ ${ }^{1}$ Osservatorio Astronomico di Trieste, Via Tiepolo 11, I-34131 Trieste, Italy \\ ${ }^{2}$ SISSA, via Beirut 4, 34014 Trieste, Italy \\ ${ }^{3}$ Dipartimento di Astronomia, Università degli Studi di Trieste, Trieste, Italy \\ E-mail: girardi, giuricin, mardiros, mezzetti, @sissa.it; boschin@newton.sissa.it
}

\begin{abstract}
We evaluate in a homogeneous way the optical masses of 170 nearby clusters $(z \leq 0.15)$. The sample includes both data from the literature and the new ENACS data (Katgert et al. 1996, 1998).

On the assumption that mass follows the galaxy distribution, we compute the masses of each cluster by applying the virial theorem to the member galaxies. We constrain the masses of very substructured clusters (about $10 \%$ of our clusters) between two limiting values.

After appropriate rescaling to the X-ray radii, we compare our optical mass estimates to those derived from X-ray analyses, which we compiled from the literature (for 66 clusters). We find a good overall agreement. This agreement is expected in the framework of two common assumptions: that mass follows the galaxy distribution, and that clusters are not far from a situation of dynamical equilibrium with both gas and galaxies reflecting the same underlying mass distribution.

We stress that our study strongly supports the reliability of present cluster mass estimates derived from X-ray analyses and/or (appropriate) optical analyses.
\end{abstract}

Subject headings: galaxies: clusters: general - galaxies: distances and redshifts - X-rays: galaxies cosmology: observations.

\section{INTRODUCTION}

The knowledge of the properties of galaxy clusters plays an important role in the study of large scale structure for'mation. In particular, the observational distribution of the abundance of galaxy clusters as a function of their mass 'places a strong constraint on cosmological models (e.g., Bahcall \& Cen 1993; Borgani et al. 1997; Gross et al. '1998; White, Efstathiou \& Frenk 1993). Moreover, recent studies stress the need for having reliable estimates of cluster masses to constrain the ratio between the baryonic to total mass and the consequent value of $\Omega_{0}$ (e.g., White \& 'Frenk 1991; White et al. 1993b).

Indeed, the estimate of cluster masses is not an easy task, in spite of the various methods which are available. The application of the virial theorem to positions and velocities of cluster member galaxies is the oldest method of cluster mass determination (e.g., Zwicky 1933). More recent methods are based on the dynamical analysis of hot X-ray emitting gas (e.g., Cowie, Henriksen, \& Mushotzky 1987; Eyles et al. 1991) and on gravitational lensing of background galaxies (e.g Grossman \& Narayan 1989).

Mass estimates derived from the dynamical analysis of gas or member galaxies which are based on the Jeans equation or its derivations, such as the virial theorem, assume that clusters are systems in dynamical equilibrium (e.g., Binney \& Tremaine 1987). This assumption is not strictly valid; in fact, although clusters are bound galaxy systems, they have collapsed very recently or are just now collapsing, as is suggested by the frequent presence of substructures (e.g., West 1994). However, some analyses suggest that the estimate of optical virial mass is robust against the presence of small substructures (Escalera et al. 1994; Girardi et al. 1997a; see also Bird 1995 for a partially different result), although it is affected by strong substructures (e.g., Pinkney et al. 1996). Similar results come from studies based on numerical simulation (e.g., Schindler 1996a; Evrard, Metzler, \& Navarro 1996; Roettiger, Burns, \& Loken 1996) for X-ray masses estimated with the standard $\beta$-model approach (Cavaliere \& Fusco Femiano 1976), although some authors have claimed there is a systematic mass underestimation (e.g.; Bartelmann \& Steinmetz 1996).

Dynamical analyses based on galaxies have the further drawback that the mass distribution or (alternatively) the velocity anisotropy of galaxy orbits should be known a priori. Unfortunately, the two quantities cannot be disentangled in the analysis of the observed velocity dispersion profile, but only in the analysis of the whole velocity distribution which, however, requires a large number of galaxies (of the order of several hundreds; e.g. Dejonghe 1987; Merritt 1988; Merritt \& Gebhardt 1994). Without some information from the relative distribution of dark and galaxy components, the virial theorem places only order-of-magnitude constraints on the total mass (e.g., Merritt 1987). The usual approach is to apply the virial theorem by assuming that mass is distributed like the observed galaxies (e.g., Giuricin, Mardirossian, and Mezzetti 1982; Biviano et al. 1993). This assumption is supported by several pieces of evidence coming both from optical (e.g., Carlberg, Yee, \& Ellingson 1997) and X-ray data (e.g., Watt et al. 1992; Durret et al. 1994; Cirimele, Nesci, \& Trevese 1997), as well as from gravitational lensing data, which, however, suggest a smaller core radius (e.g., Narayan \& Bartelmann 
1997)

The mass estimates derived from gravitational lensing phenomena are completely independent of the cluster dynamical status, but a good knowledge of cluster geometry is required in order to go from the projected mass to the cluster mass (e.g., Fort 1994). Moreover, strong lensing observations give values for the mass contained within very small cluster regions ( $\lesssim$ one hundred of $\mathrm{kpc}$ ) and weak lensing observations are generally more reliable in providing the shape of the internal mass distribution rather than the amount of mass (e.g., Squires \& Kaiser 1996).

Up to now, few studies have dealt with wide comparisons between mass estimates obtained by different methods for the same cluster. Wu \& Fang $(1996 ; 1997)$ found that masses derived from gravitational lensing analyses are higher than those from X-ray analyses by a factor of 2 , but agree with those from galaxy analyses. Indeed, mass estimates from lensing seem to agree with X-ray estimates when clusters are relaxed (e.g., Allen 1997). However, Wu \& Fang's works concern only clusters which lie at moderate redshifts and show gravitational lensing phenomena which could be enhanced in the presence of substructures (e.g., Miralda-Escudé 1993; Bartelmann, Steinmetz, \& Weiss 1995). For nearby clusters, there is a trend to obtain larger masses from galaxy analyses than from X-ray analyses (e.g., Cowie et al. 1987; Mushotzky et al. 1995; David, Jones, \& Forman 1995), but acceptable agreement exists in some individual cases (e.g., for the Coma cluster, Watt et al. 1992).

The classical approach of the virial theorem based on measurements of discrete velocities bears re-examining owing to the large new data sets which are now becoming available for nearby clusters, i.e. the ESO Nearby Abell Clusters Survey (ENACS) by Katgert et al. (1996, 1998). Moreover, the fair level of consistency among recent estimates of velocity dispersion of member galaxies resulting from different membership assignment procedures (cf. Fadda et al. 1996, hereafter F96, and Mazure et al. 1996) makes us confident of the robustness of our approach.

The aim of this work is to obtain reliable mass estimates. These mass estimates will be used in the computation of the mass function of nearby clusters (Girardi et al. 1998).

The paper is organized in the following manner. We describe the data sample and our selection procedure for cluster membership assignment in $\S 2$. We briefly describe the methods used to compute cluster masses by using member galaxies in $\S 3$. By assuming that mass follows the galaxy distribution, we compute virial mass estimates in $\S 4$, and we verify their consistency with the results of the Jeans equation in $\S 5$. The strongly substructured clusters are analyzed in $\S 6$. We compare our mass estimates with those derived from X-ray analyses in $\S 7$. We discuss our results in $\S 8$. We give a brief summary of our main results and draw our conclusions in $\S 9$.

Unless otherwise specified, we give errors at the $68 \%$ confidence level (hereafter c.l.)

A Hubble constant of $100 \mathrm{~h} \mathrm{~km} \mathrm{~s} \mathrm{~s}^{-1} \mathrm{Mpc}^{-1}$ is used throughout.

\section{THE DATA SAMPLE}

Our data sample is essentially an updating of that of F96, which is a compilation of data on nearby clusters $(z \leq 0.15)$ coming from the literature and from the new ENACS data set (Katgert et al. 1996, 1998). In this work we consider the clusters for which F96 gave an estimate of velocity dispersion. Moreover, we consider three new clusters (A3880, S639, S721) and we update the original data sets for the clusters A576, A1016, A3528N, A3556, and A3733. Table 1 lists the total cluster sample. In Col. (1) we list the cluster name; in Col. (2) the number of galaxies with measured redshift in each cluster field; in Col. (3) the Abell richness class; and in Col. (4) redshift references. The Supplementary Clusters of the ACO catalog (Abell, Corwin, \& Olowin 1989) with Abell number counts $N_{c}<30$ are classified as belonging to the richness class $R=-1$.

In order to select member galaxies, we apply the same procedure as F96 to the new (or updated) clusters. Here we summarize the main points. We use the adaptive kernel method (Pisani 1993) to find the significant peaks in velocity distributions and then the "shifting gapper" to reject galaxies that are too far in velocity (by $\geq 1000 \mathrm{~km} \mathrm{~s}^{-1}$ ) from the main body of galaxies at a given distance from the cluster center (generally within a shifting annulus of $\left.0.4 h^{-1} M p c\right)$.

Our total sample contains 170 clusters, with each cluster having at least 30 galaxies with available redshift in the original field. Ten clusters consist of two clumps obviously separated in spatial position (denoted by their spatial orientation, e.g. A548NE) or two significant peaks in the velocity distribution (denoted by "a,b,...", e.g. A151a). Eighteen clusters show a secondary peak in the velocity distribution, which strongly overlaps the primary one so that their separation is uncertain. Indeed, they could be either two different systems or two parts of the same substructured cluster and, like F96, we treat them by considering the peaks either joined or disjoined.

First we compute masses for 152 clusters which do not show ambiguity in the dynamics and allow more refined analyses, while the other 18 clusters are analyzed in $\S 6$.

\section{CLUSTER MASSES BASED ON THE DYNAMICS OF MEMBER GALAXIES}

The standard methods used to estimate the cluster mass from member galaxies require that galaxies be in equilibrium within the cluster potential. The cluster mass is then recovered from the knowledge of positions and velocities of the same population of galaxies which are taken as tracers of the cluster potential. We summarize the main points of the procedures used to estimate masses from the Jeans equation and the virial theorem. 


\subsection{THE MASS DERIVED FROM THE JEANS EQUATION}

In principle, one can estimate the cluster mass within a radius $r, M_{J}(<r)$, by using the Jeans equation, coupled with the equation which links the two observable quantities $\Sigma(R)$ and $\sigma_{P}(R)$, i.e. the projected galaxy number density and the projected velocity dispersion as a function of the projected radius $R$ :

$$
\begin{gathered}
\frac{d\left(\rho \sigma_{r}(r)^{2}\right)}{d r}+\frac{2 \rho(r) \beta \sigma_{r}^{2}}{r}=-\frac{G \rho(r) M_{J}(<r)}{r^{2}} \\
\sigma_{P}^{2}(R) \Sigma(R)=2 \int_{R}^{\infty} \rho(r) \sigma_{r}^{2}(r)\left(1-\beta \frac{R^{2}}{r^{2}}\right) \frac{r}{\sqrt{r^{2}-R^{2}}} d r
\end{gathered}
$$

where $\mathrm{r}$ is the distance from the cluster center, $\rho(r)$ is the spatial number density of galaxies linked to $\Sigma(R)$ via the Abel integral, $\sigma_{r}(r)$ is the radial component of velocity dispersion $\sigma(r)$, and $\beta(r)=1-\sigma_{\theta}^{2} / \sigma_{r}^{2}$ is the velocity anisotropy parameter (e.g., Binney \& Tremaine 1987).

Unfortunately, there are three unknowns $(M(<r), \sigma(r)$, $\beta(r)$ ) and only two equations. In order to solve these equations it is therefore necessary to make some assumptions. It seems natural to assume knowledge of either $\beta(r)$ or $M(r)$, and then to evaluate the remaining two functions so that they are consistent to the observed velocity dispersion profile (e.g., Merritt 1987).

The virial theorem derives from the Jeans equation via an integration step. It relates the global kinetic energy with the potential one $(2 T+U=0$, e.g. Binney and Tremaine 1987) and is usually used to compute virial masses.

A different derivation (Heisler, Tremaine, \& Bahcall 1985) gives an alternative mass estimator, the projected mass, which assumes an a priori velocity anisotropy parameter (see also Perea, del Olmo, \& Moles 1990 for the non-isotropic case). Since we do not use this estimator, we do not give any details about it and refer only to the original papers.

\subsection{THE MASS DERIVED FROM THE VIRIAL THEOREM}

The total virial mass of the cluster, $M_{V}$, depends on the global velocity dispersion, $\sigma$ and the spatial distribution of the galaxy population (e.g., Merritt 1988):

$$
M_{V}=\frac{<v^{2}>}{G<r^{-1} F>},
$$

where the brackets indicate spatial averages over the observed sample of $N$ galaxies, $r$ are the galaxy distances from the cluster center, and $v$ are the galaxy velocities referred to the cluster mean velocity. The function $F(r)$ is the mass fraction within $r$ and depends on the (generally) unknown form of mass distribution.

If mass is distributed like the observed galaxies (i.e., $\rho_{\text {mass }} \propto \rho$ ), then the appropriate form of eq. 3 is (Limber \& Mathews 1960):

$$
M_{V}=\frac{<v^{2}>}{G<r_{i j}^{-1}>}=\sigma^{2} R_{V} / G
$$

where $R_{V}$ is the virial radius which depends on $r_{i j}$, i.e. the distance between any pair of galaxies.

From the observational point of view, the large advantage of the virial theorem is that the global projected velocity dispersion $\sigma_{P}$ and, consequently, the total mass are independent of possible anisotropy of galaxy velocities, always being $\sigma^{2}=3 \sigma_{P}^{2}$ for spherical systems (e.g., The \& White 1986; Merritt 1988). Therefore, in the case of spherical systems, for the respective projected quantities $\sigma_{P}$ and $R_{P V}$, eq. 4 becomes:

$$
M_{V}=3 \pi / 2 \cdot \frac{<V^{2}>}{G<R_{i j}^{-1}>}=3 \pi / 2 \cdot \sigma_{P}^{2} R_{P V} / G .
$$

The uniform weighting of galaxies in computing spatial averages is largely assumed in the literature and it is justified by the absence of pronounced luminosity segregation in galaxy clusters (e.g., Adami, Biviano, and Mazure 1998; Biviano et al. 1992; Stein 1997). In this framework, the projected velocity dispersion, $\sigma_{P}$, and the projected virial radius, $R_{P V}$, are estimated as:

$$
\begin{gathered}
\sigma_{P}=\sqrt{\left(\Sigma_{i} V^{2} /(N-1)\right)}, \\
R_{P V}=N(N-1) /\left(\Sigma_{i \neq j} R_{i j}^{-1}\right) .
\end{gathered}
$$

When the system is not entirely included in the observational sample, as is usual in galaxy clusters, the usual formula of the virial theorem $2 T+U=0$ should be replaced by $2 T+U=3 P V$ where $3 P V$ is the surface term (e.g. Binney \& Tremaine 1987; Carlberg et al. 1996; Carlberg et al. 1997a; The \& White 1976). Therefore, a correction, $C$, should be applied to the usual formula of virial mass which, otherwise, overestimates the true cluster mass. In particular, if mass follows the galaxy distribution, the corrected virial mass, $M_{C V}$ is:

$M_{C V}=M_{V}-C=M_{V}\left(1-4 \pi b^{3} \frac{\rho(b)}{\int_{0}^{b} 4 \pi r^{2} \rho d r}\left(\sigma_{r}(b) / \sigma(<b)\right)^{2}\right)$,

where $\sigma(<b)$ refers to the integrated velocity dispersion within the boundary radius $b$.

\section{ESTIMATING OPTICAL CLUSTER MASSES}

In this section we compute the cluster masses on the hypothesis that galaxy number-distribution traces mass distribution. Therefore, the results of $\S 4.5$ and 4.6 depend on this assumption.

From the observational point of view, compared to use of the Jeans equation, the virial theorem has the great advantage of using the more robust integrated values of $\sigma_{P}$ rather than the differential values. Since the computation 
of the observational dispersion profile $\sigma_{P}(R)$ requires a large number of galaxies, we can compute it only by combining together the data of many clusters, without preserving cluster individuality. Therefore, we use the virial theorem to compute the mass of each individual cluster and then we use the Jeans equation to check our results on "average" clusters $(\S 5)$.

\subsection{VELOCITY DISPERSION}

The problems concerning the estimate of the velocity dispersion, $\sigma_{P}$, have been faced in two previous papers (F96; Girardi et al. 1996). These studies found that the observed profiles $\sigma_{P}(<R)$, where the velocity dispersion is computed by including larger and larger cluster regions, may show strongly increasing (or decreasing) behaviors in the central cluster regions, but flatten out in the external regions, suggesting that in these regions the (almost constant) value of $\sigma_{P}$ is no longer affected by possible velocity anisotropies. Therefore, in eq. 5 one can consider as physically meaningful only the value of $\sigma_{P}$ computed by considering all galaxies within a large radius.

We use the values of projected velocity dispersions as tabulated in Table 3 of F96 and, for new (and updated) clusters, we adopt their same procedure to estimate $\sigma_{P}$. In particular, we use robust estimates (computed via the ROSTAT routines by Beers, Flynn, \& Gebhardt 1990), obtained by applying the relativistic correction and the usual correction for velocity errors (Danese, De Zotti, \& di Tullio 1980).

\subsection{THE RADIUS OF VIRIALIZATION}

Since the application of the virial theorem is really meaningful only when the system is in dynamical equlibrium within the region considered, the natural choice is to compute cluster masses within the radius of virialization, $R_{v i r}$. The precise computation of the radius of virialization is possible only after the computation of cluster mass $(\S 4.6)$ and would require a recursive procedure. This will be given in Girardi et al. (1998) for a few standard cosmological scenarios. Here, we give a reasonable a priori approximation.

In the framework of the theory of a spherical model for nonlinear collapse, the relation between the present density of a collapsed (virialized) region and the present cosmological density is $\rho_{v i r}\left(t_{0}\right)=18 \pi^{2} \rho_{0}=18 \pi^{2} \times 3 H_{0}^{2} / 8 \pi G$ (for a $\Omega_{0}=1$ Universe). As a first approximation, the virial mass $\left(M_{v i r}=4 \pi R_{v i r}^{3} \rho_{v i r} / 3\right)$ can be estimated as $3 \pi / 2 \times \sigma_{P}^{2} R_{P V} / G$ (eq. 5). Therefore:

$$
R_{v i r}^{3}=\sigma_{P} R_{P V} /\left(6 \pi H_{0}^{2}\right)
$$

where the projected virial radius, $R_{P V}$, depends on the galaxy distribution.

From observed galaxy distributions, Girardi et al. 1995 (hereafter G95; see eqs. A6,A8) showed that the typical $R_{P V}$ is related to the aperture $A$, i.e. the radius of the sampled region (here equal to $R_{v i r}$ ), as follows:

$$
R_{P V}=1.193 A \frac{1+0.032\left(A / R_{c}\right)}{1+0.107\left(A / R_{c}\right)},
$$

where the core radius $R_{c}$ is on average $=0.17$ $h^{-1}$ Mpc (G95). Eqs. 9 and 10 imply the following relation between $R_{v i r}$ and $\sigma_{P}$ :

$$
R_{v i r} \sim 0.002 \cdot \sigma_{P}\left(h^{-1} M p c\right),
$$

with $\sigma_{P}$ given in $k m \mathrm{~s}^{-1}$.

In the following analyses we consider only member galaxies within $R_{v i r}$.

\subsection{GALAXY DISTRIBUTION}

We examine the galaxy distribution of the 92 wellsampled clusters, i.e. the clusters sampled with at least ten galaxies up to $R_{v i r}$. Their galaxy distribution are analyzed in a similar way to that used by G95, i.e. by fitting the galaxy surface density of each cluster to a King distribution with a variable exponent (hereafter referred to as a "King-like" profile):

$$
\Sigma(R)=\frac{\Sigma_{0}}{\left(1+\left(R / R_{c}\right)^{2}\right)^{\alpha}},
$$

where $R_{c}$ is the core radius and $\alpha$ is the parameter which describes the galaxy distribution in external regions $(\alpha=1$ corresponds to the classical King distribution). This surface density profile corresponds to a galaxy volume-density $\rho=\rho_{0} /\left(1+\left(r / R_{C}\right)^{2}\right)^{3 \beta_{f i t, g a l} / 2}$, with $\beta_{f i t, g a l}=(2 \alpha+1) / 3$, i.e. $\rho(r) \propto r^{-3 \beta_{f i t, g a l}}$ for $r>>R_{C}$. We perform the fit through the Maximum Likelihood technique, allowing $R_{C}$ and $\alpha$ to vary from 0.01 to 1 and from 0.5 to 1.5 , respectively, and verifying that the fitted profiles are not rejected by a Kolmogorov-Smirnov test.

In order to explore the typical galaxy distribution, we re-do the fits by fixing the value of $\alpha$, the less scattered parameter (see also G95). The median value of $\alpha$, with the respective $90 \%$ c.l. intervals, is $=0.70_{-0.03}^{+0.08}$, corresponding to a $\beta_{\text {fit,gal }}=0.8$, i.e. to a volume galaxy-density $\rho \propto r^{-2.4}$. By fixing the value of $\alpha$, we again fit the galaxy distribution of each cluster, obtaining median values of $R_{c}=0.05_{-0.01}^{+0.01} h^{-1} M p c(90 \%$ c.l. intervals) and a $R_{c} / R_{\text {vir }}=0.05$.

As a final check, we analyze the effect of galaxy incompleteness in the external cluster regions, which could mimic a greater value of $\alpha$. We consider a subsample of 27 clusters (denoted by an asterisk in Table 1) from which it is possible to extract magnitude-complete samples (at least up to the $80 \%$ completeness level) according to the prescriptions of the authors of redshift data. After having performed the two-parameter fit, we find a median value of $\alpha=0.65_{-0.07}^{+0.05}$ (90\% c.l. intervals), in agreement with the value obtained from the whole cluster sample.

\subsection{THE VIRIAL RADIUS}

The projected virial radius $R_{P V}$ depends on galaxy distribution and increases with the cluster aperture $A$ within 
which it is estimated. Here, we are interested in determining $R_{P V}$ at $A=R_{v i r}$. We cannot use the direct estimate of $R_{P V}$ (eq. 7 ) for the clusters that are not sampled at least up to $R_{v i r}$ (these 50 clusters are sampled up to $R_{\max }=0.7 \times R_{v i r}$, on average) or have too small a number of galaxies.

Once the values of $R_{C}$ and $\alpha$ are known, we can use the alternative estimate based on the galaxy distribution to compute $R_{P V}$ at $R_{v i r}$ (eq. A1 of G95). We consider three estimates $\left(R_{P V}\left(\alpha, R_{C}\right), R_{P V}\left(\alpha=0.7, R_{C}\right)\right.$, and $\left.R_{P V}\left(\alpha=0.7, R_{C}=0.05 \times R_{v i r}\right)\right)$, respectively based on: a) the values of $\alpha$ and $R_{C}$ resulting from the above twoparameter fit, b) the median value of $\alpha$ and the value of $R_{C}$ resulting from the one-parameter fit, and c) the median values of $\alpha$ and $R_{c}$. In particular, we note that for $\alpha=0.7$, the relation between $R_{P V}$ and $R_{C}$ for a given cluster aperture $A$ (eq. 1 of G95) is well represented by:

$$
R_{P V}=1.189 A \frac{1+0.053\left(A / R_{c}\right)}{1+0.117\left(A / R_{c}\right)}
$$

We analyze the 92 well-sampled clusters, which are sampled up to $R_{v i r}$, to verify the acceptability of the three alternative procedures. Figure 1 (top-left panel) shows that the virial radius estimated from the individual fitted values of $R_{C}$ and $\alpha, R_{P V}\left(\alpha, R_{C}\right)$, is a good alternative estimate of the direct estimate of $R_{P V}$. When one or two parameters are fixed, the estimates result in larger and larger scatter in the relation with $R_{P V}$ (see Figure 1, topright and bottom-left panels, respectively), supporting the existence of an intrinsic spread of cluster parameters.
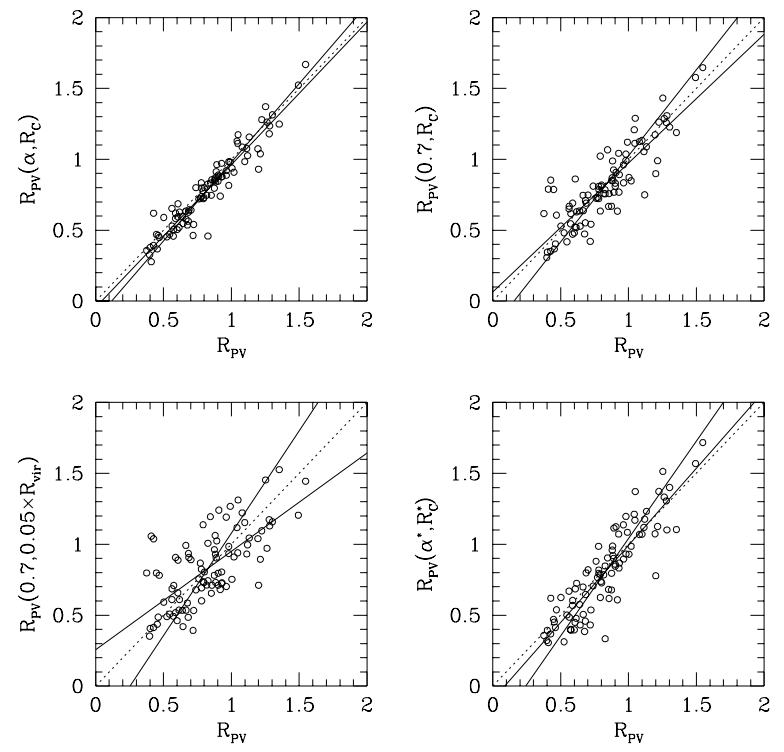

Fig. 1. - Four alternative estimates of the virial radius $R_{P V}$ obtained from galaxy distribution parameters $\left(\alpha, R_{C}\right)$ vs. the direct estimate obtained from galaxy separations. The units are in $h^{-1} M p c$. The solid lines represent the linear fits (y vs. $\mathrm{x}$ and $\mathrm{x}$ vs. $\mathrm{y}$ regression lines).
Moreover, in order to check the effect of the limited cluster aperture, we also compute the estimate $R_{P V}\left(\alpha^{*}, R_{C}^{*}\right)$ by using $R_{C}$ and $\alpha$ fitted within $0.7 \times R_{v i r}$ rather than within $R_{v i r}$. The values of $R_{P V}$ are recovered by a larger scatter with respect to the optimal case (cf. bottom-right panel with top-left panel in Figure 1).

Summarizing, we compute $R_{P V}$ (at $R_{v i r}$ ): i) by using the direct definition of eq. 7 for 100 clusters; ii) by using the estimate obtained from the fitted values of $\alpha$ and $R_{C}$ for the 50 clusters with $R_{\max }<R_{\text {vir }}$; iii) by using the estimate obtained from the typical values of $\alpha=0.7$ and $R_{C}=0.05 \times R_{v i r} h^{-1} M p c$ only for 8 clusters with a very small number of galaxies $(\leq 6)$. For case i), i.e. the direct estimate, we compute the jackknife error; in cases ii) and iii) we adopt the respective s.d. of residuals from the direct regression line, i.e. 0.15 and $0.19 h^{-1} \mathrm{Mpc}$.

The effect of cluster asphericity on the estimate of $R_{P V}$ may be estimated directly if one assumes that galaxy distributions are axisymmetric (prolate or oblate), and that isodensity surfaces are concentric, similar ellipsoids. We use Monte Carlo simulations to evaluate the projection factor, $f_{R}=R_{V} / R_{P V}$, as a function of the inclination and of the intrinsic shape. In particular, we simulate the galaxy systems by requiring that, along each axis, the particles fit a 1-D King distribution with a core radius proportional to the cluster dimension along that axis and we compute a mean value of $f_{R}$ by averaging 200 simulated systems of 100 particles for each one. Even if $f_{R}$ can strongly differ from the spherical symmetric value (i.e., $\pi / 2$ ), its expectation value is $1.58 \pm 0.19$, if we assume that clusters are prolate and have a Gaussian distribution of intrinsic axial ratios with a mean of 0.5 and a s.d. of 0.15 (Plionis, Barrow, \& Frenk 1991). Moreover, we have verified that Gaussian or uniform particle distributions give similar mean $f_{R}$ values to those obtained for a King-distribution. Owing to large errors involved in mass computations, we see no reason to adopt a projection factor different from $\pi / 2$.

\subsection{VELOCITY ANISOTROPIES}

In order to compute the $C$-correction to the virial mass (eq. 8) we must address the topic of velocity anisotropies of galaxy orbits.

After having assumed the velocity anisotropy parameter $\beta(r)$, one can compute $\sigma_{r}(r)$ by solving the Jeans equation (eq. 1) and then obtain the projected velocity dispersion $\sigma_{P}(R)$ (eq. 2) which can be compared with the data. The observational $\sigma_{P}(R)$ is obtained by combining together the galaxies of many clusters, i.e. by normalizing distances to $R_{v i r}$ and velocities (relative to the mean cluster velocity) to the observed global velocity dispersion $\sigma_{P}$.

The simplest approach is to assume that velocities are isotropic, i.e. $\beta(r)=0$. Figure 2 shows the observed profile $\sigma_{P}(R)$, as obtained by joining together all clusters, plotted against the theoretical profiles obtained by using the typical galaxy distribution ( $\S 4.3)$. The model is compatible with our data at the $96 \%$ c.l..

Den Hartog \& Katgert (1996) and F96 show that dif- 
ferent clusters have markedly different velocity dispersion profiles. This suggests that the flatness of combined profile is due to the mixing of clusters with different kinds of velocity anisotropies and, therefore, with different $C$ corrections. In order to give more realistic corrections for individual clusters, we decide to divide clusters according to the shape of their profiles.

We take as a profile indicator, $I_{p}$, which is the ratio between $\sigma_{P}\left(<0.2 \times R_{v i r}\right)$, the velocity dispersion computed by considering the galaxies within the central $0.2 \times R_{v i r}$, and the global $\sigma_{P}$. The data are good enough to compute $I_{p}$ for 136 systems. Note that the individual shape of velocity-dispersion profiles $\left(I_{p}\right)$ does not correlate with cluster velocity dispersion (see also den Hartog \& Katgert 1996). We divide clusters in to three equivalent groups of 45, 46, 45 clusters (hereafter A, B, C clusters, going from high to low $I_{p}$, respectively) and build three observational profiles $\sigma_{P}(R)$, which are decreasing, flat and increasing with $R$, respectively.

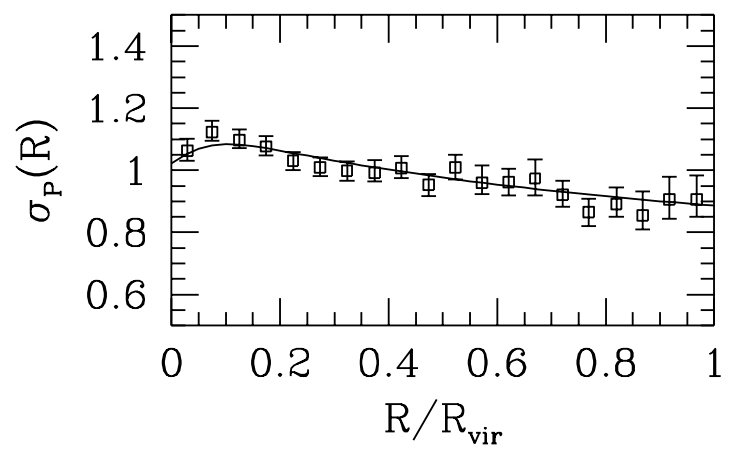

Fig. 2.- The (normalized) projected velocity dispersion, $\sigma_{P}$, as a function of the (normalized) projected distance from the cluster center. The points represent data combined from all clusters and binned in equispatial intervals. We give the robust estimates of velocity dispersion and the respective bootstrap errors. The solid line represents the model for the constant velocity anisotropy parameter $\beta=0$.

In order to fit the profiles of $\mathrm{A}$ and $\mathrm{C}$ clusters, we introduce two other functional forms for $\beta(r)$. The form $\beta=r^{2} /\left(r^{2}+a^{2}\right)$ (e.g., Merritt 1987) describes a cluster with isotropic velocities in the center and radial velocities in the external regions, as expected in the case of galaxy infall from the regions around the cluster; this form gives a decreasing profile (A clusters). We use the simple form $\beta=-c / r$ for describing circular velocities in the center and more isotropic velocities in the external regions, as expected for a very relaxed cluster undergoing two-body relaxation in the dense central region; this form gives an increasing profile (C clusters).

We compute theoretical profiles for the cases A, B, C as in the case of all clusters together. Figure 3 shows the observed profiles for the three categories of clusters, each one plotted against the best theoretical profile. In particular, the fit (acceptable at $\sim 2 \%$ s.l.) for A clusters corresponds to $\beta(r)=r^{2} /\left(r^{2}+a^{2}\right)$ with $a=0.1 \times R_{v i r}$; the fit (acceptable at $\sim 60 \%$ s.l.) for B clusters corresponds to $\beta(r)=0$; the fit (acceptable at $\sim 6 \%$ s.l.) for $\mathrm{C}$ clusters corresponds to $\beta(r)=-c / r$ with $c=1 \times R_{\text {vir }}$.
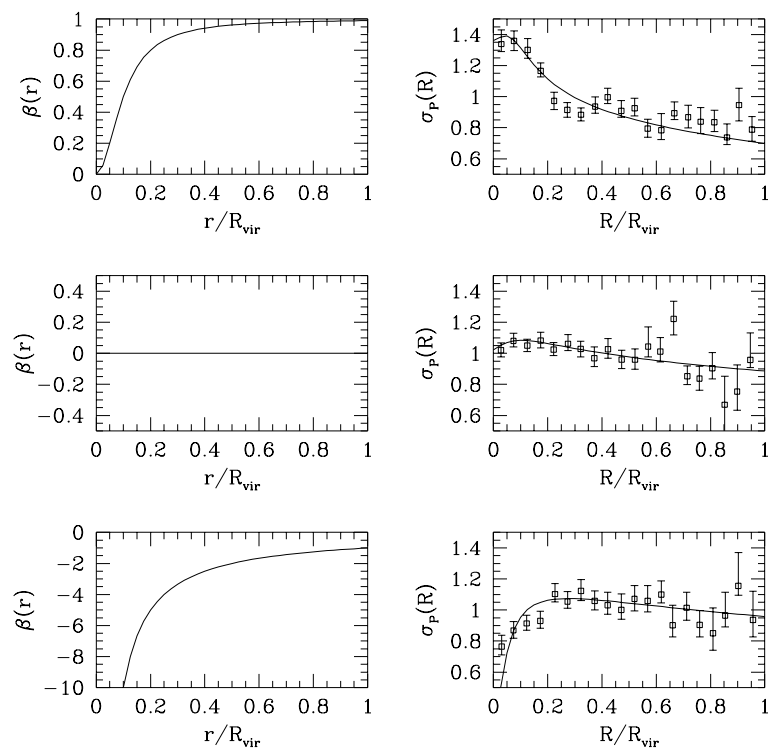

Fig. 3.- From top to bottom: we refer to clusters of type "A", "B", and "C". At right, the (normalized) projected velocity dispersion, $\sigma_{P}$, as a function of the (normalized) projected distance from the cluster center, as in Figure 2. But, here, the solid line represents the model for the velocity anisotropy parameter $\beta(r)$ shown in the corresponding panel on the left.

We note that, in this study, which is not devoted to the analysis of velocity anisotropies, the simple forms adopted for $\beta(r)$ are adequate for giving a satisfactory estimate of the $C$-correction. For instance, when we analyze the whole cluster sample by using the two other functional forms for $\beta(r)$ (instead of $\beta(r)=0$ ) and requiring a compatibility of the fit at only $\sim 2 \%$ c.l., we find that the two respective $C$-corrections differ by $\sim 25 \%$, corresponding to a variation for the mass of $\sim 5 \%$ (well within the associated observational errors, see $\S 4.6$ ).

\subsection{THE VIRIAL MASS}

In Table 2 we list the cluster parameters computed before: the number of member galaxies, $N_{m}$ (Col. 2), contained within the radius $R_{\max }$ (Col. 3) and used to compute the mean (galactocentric) redshift (Col. 4) and the global projected velocity dispersion $\sigma_{P}$ with the respective bootstrap errors (Col. 5); the number of galaxies, $N$ (Col. 6), within $R_{v i r}$ (Col. 7); the values of $\alpha$ and $R_{C}$ resulting from the fit to the galaxy distribution (Cols. 8 and 9 , respectively); the projected virial radius, $R_{P V}$, computed at $R_{v i r}$ (Col. 10); the cluster type according to their velocity dispersion profile, $T$ (Col. 11).

By using $\sigma_{P}$ and $R_{P V}$, we compute the virial mass $M_{V}$ within $R_{v i r}$ through the standard formula (eq. 5).

However, clusters are well extended outside the virial radius $R_{v i r}$. By using the theoretical expressions of Gunn 
\& Gott (1972) and the same procedure as that described in $\S 4.2$, we find that the radius of turn-around and the radius of the region gravitationally bound to the cluster are $\sim 5-10 R_{v i r}$. Therefore, we need to apply the $C$-correction (eq. 8) to obtain the corrected virial mass $M_{C V}$. Here we re-write the $C$-correction of eq. 8 by taking into account the fact that the boundary radius is $b=R_{\text {vir }}$ :

$$
C=M_{V} 4 \pi R_{v i r}^{3} \frac{\rho\left(R_{v i r}\right)}{\int_{0}^{R v i r} 4 \pi r^{2} \rho d r}\left(\sigma_{r}\left(R_{v i r}\right) / \sigma\left(<R_{v i r}\right)\right)^{2},
$$

The term $\rho\left(R_{v i r}\right) / \int_{0}^{R v i r} 4 \pi r^{2} \rho d r$ is computed for each cluster by using the the galaxy distribution given in eq. 12 with the appropriate individual parameters.

The term $\left(\sigma_{r}\left(R_{v i r}\right) / \sigma\left(<R_{v i r}\right)\right)^{2}$ is connected to the presence of velocity anisotropies analyzed in the above subsection.

For all clusters joined together, in the case of best fit with the data $(\beta=0$; see Figure 2$)$, we find that $\left(\sigma_{r}\left(R_{v i r}\right) / \sigma\left(<R_{v i r}\right)\right)^{2}$ is $\sim 0.3$. For the A, B, C clusters with different kinds of profiles (see Figure 3 ), the corresponding values of $\left(\sigma_{r}\left(R_{\text {vir }}\right) / \sigma\left(<R_{\text {vir }}\right)\right)^{2}$ are $\sim$ $0.6,0.3,0.2$, respectively. We adopt the above values of $\left(\sigma_{r}\left(R_{\text {vir }}\right) / \sigma\left(<R_{\text {vir }}\right)\right)^{2}$ for $\mathrm{A}, \mathrm{B}, \mathrm{C}$ clusters and the value obtained for the whole sample (0.3) for 24 other clusters which we cannot assign to any specific kind of profile.

The median value of percentage $C$-correction is $19 \%$ and rises to $39 \%$ when we consider clusters with a decreasing profile towards external regions (clusters of type A).

In Table 3 we list the virial masses, within $R_{v i r}$, before and after the correction, $M_{V}$ and $M_{C V}$ (Cols. 2 and 3, respectively). We assume that the percentage errors on $M_{C V}$ are the same as for $M_{V}$, i.e. neglecting uncertainties on the $C$-correction.

In conclusion, we stress the fact that in the computation of the $\left(\sigma_{r}\left(R_{v i r}\right) / \sigma\left(<R_{v i r}\right)\right)^{2}$ term, we have implicitly assumed that the region outside $R_{v i r}$ is in dynamical equilibrium, too. Since there is no evidence of a drastic change of profiles of galaxy density and velocity dispersion at $R_{v i r}$ (see, e.g., Fig. 6 of Kent \& Gunn 1982; Fig. 2 of F96), we can regard our estimate of $C$-correction as a good first approximation. The analysis of $\S 5$ will show that our estimate is qualitatively and quantitatively acceptable.

\section{VERIFYING THE SELF-CONSISTENCY OF MASS ESTIMATES}

The virial theorem and the Jeans equation should give consistent mass estimates. Owing to the typically limited number of member galaxies for each individual cluster, the Jeans equation is not useful for constraining individual cluster masses, but can be useful for checking average results.

As a first approximation, cluster galaxies can be described, on average, by an isothermal distribution, i.e by having a constant $\sigma$-profile, and isotropic velocities (see $\S 4.5)$. Consequently, by using the Jeans equation, one expects that the cluster mass contained within a large radius $r$ is $M_{i s o}(<r)=3 \beta_{f i t, g a l} \sigma_{P}^{2} r / G$ (here $\beta_{f i t, g a l}=0.8$; see $\S 4.3)$. We compute $M_{\text {iso }}\left(<R_{\text {vir }}\right)$ for each cluster. In Figure 4 we show $M_{\text {iso }}\left(<R_{\text {vir }}\right)$ against $M_{V}$ and $M_{C V}$, respectively. We find that $M_{i s o}$ values are smaller than $M_{V}$, the virial masses before the $C$-correction, but are roughly consistent with $M_{C V}$.
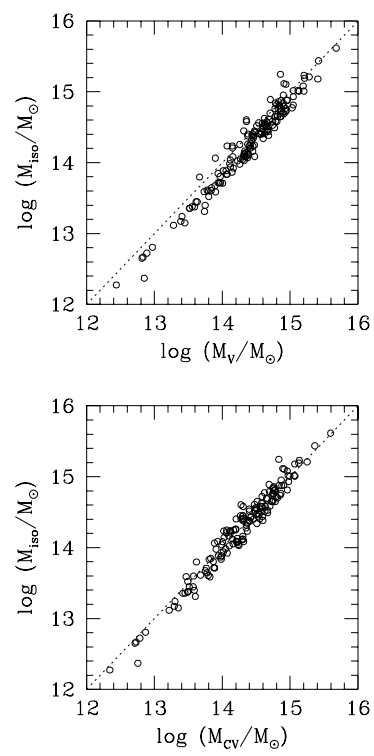

Fig. 4.- The comparison between the mass obtained from the Jeans equation by assuming a constant $\sigma$-profile and isotropic velocities vs. the virial mass before (upper) and after (lower) the $C$ correction.

In order to explore the results for clusters with different velocity anisotropies, we also consider another approach. Retaining the hypothesis that mass follows the galaxy distribution, one can obtain cluster mass estimates from the Jeans equation, $M_{J}$, without any further hypothesis $(\S 3.1)$. Indeed, by applying the same procedure as in $\S 4.5$, but constraining $\sigma_{P}\left(<R_{\text {vir }}\right)$ to be equal to the typical (median) observational value, we obtain a value for the normalization of mass. In Table 4 we give for each type of clusters (A, B, C): in Col. (2) the number of clusters belonging to the corresponding type; in Cols. (3) and (4) the value of observed $\sigma_{P}$ and the corresponding $\log \left(M_{J}\left(<R_{v i r}\right)\right)$ computed from the Jeans equation; in Cols. (5) and (6) the average values of $\log \left(M_{V}\right)$ and $\log \left(M_{C V}\right)$ as computed in the above subsection. Again the value of $M_{J}$ turns out to be smaller than $M_{V}$, but in fair agreement with $M_{C V}$.

The general result of our check is that a correction to the standard virial masses, $M_{V}$, is really needed and that the $C$-correction appears to be a good estimate of this correction. 


\section{STRONGLY SUBSTRUCTURED CLUSTERS}

The strongly overlapping peaks shown by 18 of our clusters in their velocity distribution may have several explanations. The peaks may be different systems superimposed along the line of sight, maybe before a merging, or could indicate the presence of substructures in a single system. Remarkably, merging galaxy clumps can also survive the first encounter (McGlynn \& Fabian 1984; Roettiger, Stone, \& Mushotzky 1998), making it more difficult to understand their real dynamical state. Indeed, the understanding of the dynamics of these clusters requires individual analysis, with many more data for each cluster.

Here we limit our analysis to constraining the masses of these 18 clusters between two limiting values, obtained by considering the peaks to be either joined or disjoined. We compute these masses by applying the same procedure as that used above. The respective cluster parameters and cluster masses are listed in Tables 5, 6, and 7,8 which have the same format as Tables 2 and 3, respectively.

The mass estimates strongly depend on the treatment we choose. We find that the masses obtained in the case of joined peaks differ from those obtained in the case of the disjoined peaks (by adding the values of the individual peaks) by a factor of three, on average.

\section{THE COMPARISON WITH X-RAY MASSES}

From the literature, we collect 117 values of X-ray masses, $M_{X}$, for 66 clusters. When a reference source presents the value of $M_{X}$ at several radii, we choose the value corresponding to the largest radius, avoiding possible extrapolation outside the observed X-ray region. We avoid strongly substructured clusters, for which the X-ray analysis may also be problematic (e.g., Roettiger et al. 1996). This collection of $M_{X}$ is quite inhomogeneous as regards the method of analysis, source of X-ray data, cluster richness, and observational aperture.

In Table 9 we list: the source of the X-ray masses (Col. 2); the value of X-ray radius (Col. 3), $R_{X}$, within which $M_{X}$ (Col. 4) is computed; our optical masses $M_{o p t}$ (Col. 5) obtained by rescaling each $M_{C V}$ to $R_{X}$ by using the individual galaxy distribution. When the authors did not give errors for $M_{X}$, we adopt the percentage error on X-ray temperature $T$, if available, or, otherwise, a bona fide error of $50 \%$. For $M_{o p t}$ we adopt the same percentage errors as for $M_{C V}$.

In Figure 5 we show the comparison between $M_{X}$ and $M_{\text {opt }}$. We distinguish the data by White, Jones \& Forman (1997), which represent a large subsample characterized by homogeneity in the method of analysis and the source of X-ray data.

The masses of individual clusters do not agree in several cases. The quality and amount of difference depend not only on the cluster examined, but also on the reference sources of $M_{X}$ (e.g., for the Coma cluster), as expected in an inhomogeneous sample. The two points which show the largest discrepancies refer to AWM4 cluster which, al- though defined as a poor cluster, shows a very high X-ray temperature of $3.7 \mathrm{keV}$ (e.g., David et al. 1993 corresponding to $\sigma_{P} \sim 800 \mathrm{~km} \mathrm{~s}^{-1}$ on the hypothesis of density energy equipartition between gas and galaxies) when compared with the observed velocity dispersion (about 100 $\mathrm{km} / \mathrm{s}$ ). This cluster could be a case of merging between clumps and, therefore, could be farther from a situation of dynamical equilibrium; however, present galaxy sampling is so poor that we cannot be more conclusive.

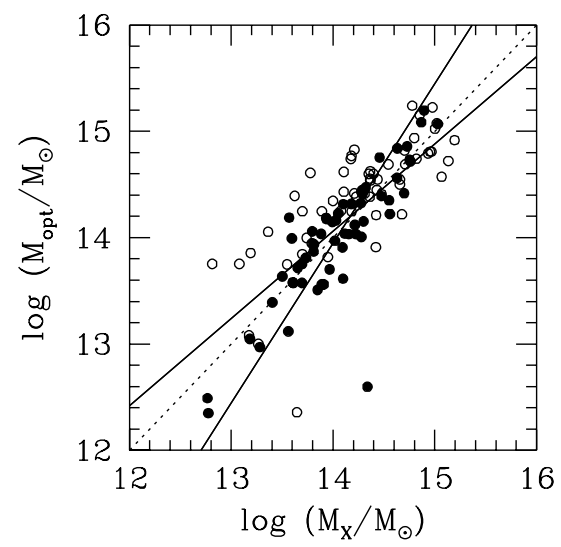

Fig. 5. - The comparison between our optical mass estimates and those derived from X-ray analysis. The optical masses are obtained by appropriately rescaling our corrected virial masses to the same radius as that the X-ray masses refer to. The solid lines represent the linear fits ( $\mathrm{y}$ vs. $\mathrm{x}$ and $\mathrm{x}$ vs. $\mathrm{y}$ regression lines). The solid points represent the data by White et al. (1997).

The overall agreement is good. The bisecting regression line and the weighted regression line (Press et al. 1982) are $M_{o p t}=10^{(-1 \pm 1)} \times M_{X}^{(1.11 \pm 0.07)}$ and $M_{o p t}=$ $10^{(0.4 \pm 0.4)} \times M_{X}^{(1.01 \pm 0.03)}$, respectively. The s.d. of residuals from the weighted regression line gives a typical deviation of mass values of $\sim 30 \%$. Part of this scatter is probably due to the inhomogeneity of the X-ray data compilation. In fact, by considering only the data by White et al. (1997) we reduce the deviation of mass values to $\sim 25 \%$. According to the $\chi^{2}$ fit probability, the observed scatter cannot be explained only by the errors on masses unless the percentage errors are underestimated by a factor of two.

\section{DISCUSSION}

We find a fair average agreement between our optical masses and X-ray masses.

The previous tendency of finding optical estimates larger than X-ray estimates (Mushotzky et al. 1995; David et al. 1995) can be explained by the fact that our mass estimates are generally smaller than the previous ones. After appropriate rescaling, we compared our estimates with those of other studies with some clusters in common. We consider 
the optical mass estimates by Biviano et al. (1993; 56 clusters), Bird (1995; 18 clusters), and Girardi et al. (1997a; 8 clusters) finding that our estimates are smaller by $40 \%$, $40 \%$, and 20\%, respectively. The difference with Biviano et al. is partially due to our more rigorous rejection of interlopers. The remaining difference and the difference with Girardi et al. are explainable by our application of the $C$-correction (due to the surface term in the virial theorem).

The difference with Bird's (1995) estimates is due to a combination of the following effects: i) a different rejection of interlopers (our $\sigma_{P}$ are smaller by $7 \%$ than Bird's values, leading to a difference in the masses by a factor of $14 \%$ ); ii) the effect of the different mass estimator; iii) the effect of the $C$-correction. Bird (1995) used the projected mass estimator, which assumes that mass follows galaxy distribution and that galaxy velocities are isotropic, whereas the virial estimator does not require any assumption about velocity anisotropies. In the case of radial or circular anisotropies, the virial estimator gives, respectively, larger or smaller mass estimates that the projected estimator (e.g. Perea et al. 1990). However, this different behavior is counterbalanced by the effect of the $C$ correction which reduces virial masses by a large amount in the case of radial orbits (clusters of type $\mathrm{A}$ in $\S 4.5$ and 4.6) and by a small amount in the case of isotropic and circular orbits (clusters of types B and C). Given these two combined effects, for both A clusters and B $+\mathrm{C}$ clusters we obtain masses which differ from Bird's estimates by a similar fraction (note that we have 10:5:3 clusters of types $\mathrm{A}: \mathrm{B}: \mathrm{C})$.

Our estimate of $C$-correction (19\%, median value) is in good agreement with that given by The \& White (1976) for the Coma cluster (27\%, the maximum possible correction) and with that recently suggested by Carlberg et al. (1997a, 18\% mean value for CNOC clusters). We stress the fact that our estimate of this correction is only an approximation for real clusters, since it assumes that the cluster is entirely in dynamical equlibrium and not only up to the virialization radius. The overall reliability of this mass correction is supported a posteriori by the fair agreement between the corrected virial mass and the mass derived from the Jeans equation. Moreover, a quantitatively similar correction is obtained by using an alternative approach which analyzes the effect of galaxy infall and turbulent galaxy motions around clusters on observational cluster parameters (Mezzetti et al. 1998).

The agreement between optical and X-ray masses is well explained in the context of two common assumptions: i) that mass follows the galaxy distribution, ii) that clusters are not far from a situation of dynamical equilibrium, with both gas and galaxies reflecting the same underlying mass distribution.

Both our estimates of virial masses and the verification with the Jeans equation are based on the assumption that mass follows the galaxy distribution, whereas X-ray masses do not require any a priori assumption about the cluster mass distribution. Therefore, the agreement we find between $M_{o p t}$ and $M_{X}$ provides a good support for the "mass follows galaxies" assumption. In particular, in order to look for possible departures from this law, we also analyze the relation between the ratio $M_{X} / M_{o p t}$ and $R_{X}$ ( $R_{X}$ ranging from 0.06 to $\sim 2 \times R_{v i r}$ ), finding no significant correlation. Our results regarding mass distribution are to be added to considerable evidence coming from optical, X-ray analyses, and gravitational lensing (e.g., Carlberg et al. 1997a; Cirimele et al. 1997; Durret et al. 1994; Narayan \& Bartelmann 1997; Watt et al. 1992).

The fact that gas should be roughly in dynamical equilibrium within clusters is shown by several works based on numerical simulations (e.g., Schindler 1996a; Evrard et al. 1996). As regards galaxies, by using CNOC cluster data, Carlberg et al. (1997b) have recently found that both red and blue galaxy populations are in dynamical equilibrium within the cluster potential.

Our relation between optical and X-ray mass estimates shows a large scatter which cannot be explained by the observational errors (unless they are strongly underestimated). This fact suggests the presence of an intrinsic scatter, possibly due to some deviation from the "mass follows galaxies" law or from pure dynamical equilibrium.

Moreover, we do not rule out that there may be some clusters which are far from dynamical equilibrium and, indeed, we cannot make any analysis for our strongly substructured clusters whose mass value is very uncertain (Tables 7 and 8). Therefore, our conclusion is that about $90 \%$ of nearby clusters are not far from dynamical equilibrium, in agreement with the results of a statistical analysis devoted to cluster substructures in 48 clusters (Girardi et al. 1997a). For clusters which lie at moderate or large distances, the question is still open since evidence both in favor of and against dynamical equilibrium has been reported (e.g., Allen 1997; Allen, Fabian, \& Kneib 1996; Carlberg et al. 1997a; Girardi et al. 1997b; Loeb \& Mao 1994; Miralda-Escudé \& Babul 1995; Wu 1994).

On the assumptions of cluster dynamical equilibrium, we can further investigate the relation between galaxies and gas components. As a first approximation, one can assume that the hot gas is isothermal, and that galaxies have a constant velocity dispersion profile and isotropic orbits. On these assumptions, the Jeans equation gives $\beta_{\text {spec }}\left(:=\sigma_{P}^{2} \mu m_{p} / k T\right)=\beta_{\text {fit,gas }} / \beta_{\text {fit,gal }}$ where $\beta_{\text {fit }}$ gives the slope of the density distribution in external cluster regions, $T$ is the gas temperature, $\mu$ is the mean molecular weight and $m_{p}$ the proton mass (e.g., Gerbal, Durret, Lachiéze-Rey 1994; Bahcall \& Lubin 1994). Since the gas distribution is more extended than the galaxy distribution $\beta_{\text {fit,gas }}=0.67$ (e.g., Jones \& Forman 1984) against $\beta_{f i t, \text { gal }}=0.8$ as found in this work (see also Bahcall \& Lubin 1994 and references therein), we expect that the gas is "hotter" than the galaxies.

Indeed, by comparing our $\sigma_{P}$ with $T$ taken from David et al. (1993) and from White et al. (1997), we obtain that $\beta_{\text {spec }}=0.88 \pm 0.04$. Note that this value refers to 55 clusters with better optical data according to the requirements of Girardi et al. (1996), i.e. with at least 30 member 
galaxies and excluding strongly structured clusters. The fitted weighted relation is $\sigma_{P} \propto 10^{2.51 \pm 0.03} \times T^{0.62 \pm 0.04}$ (see Figure 6) which is consistent with that given in Girardi et al. (1996) but no longer consistent with the model of perfect galaxy/gas equipartition $\left(\beta_{\text {spec }}=1\right)$. This relation suggests some dependence of $\beta_{\text {spec }}$ on cluster mass (see also Bird, Mushotzky, \& Metzler 1995).

The above phenomena can be explained on the view that galaxies are slowed down by dynamical friction (e.g. Bird et al. 1995) and/or that gas is heated by galactic winds (e.g., White 1991) or during a preheating phase of the intracluster medium (e.g., Cavaliere, Menci, \& Tozzi 1997). Recent studies based on numerical simulations, even when galactic winds are included (e.g., Metzler \& Evrard 1997), suggest that the effect on the galaxies is the main one, since they found the presence of a velocity bias, i.e. galaxies slowed down with respect to dark matter, $D M$. But in this scenario the $D M$ distribution is more extended than the galaxy distribution, hence virial masses should strongly underestimate cluster masses (by up to a factor of 5, according to Frenk et al. 1996). However, these results on DM distributions are in disagreement with the results of X-ray analyses (e.g., Cirimele et al. 1997; Durret et al. 1994; Watt et al. 1992) which, instead, give evidence of a DM distribution less extended than that of gas as expected in the case of heating of the gas to temperatures higher than those of galaxies and DM.

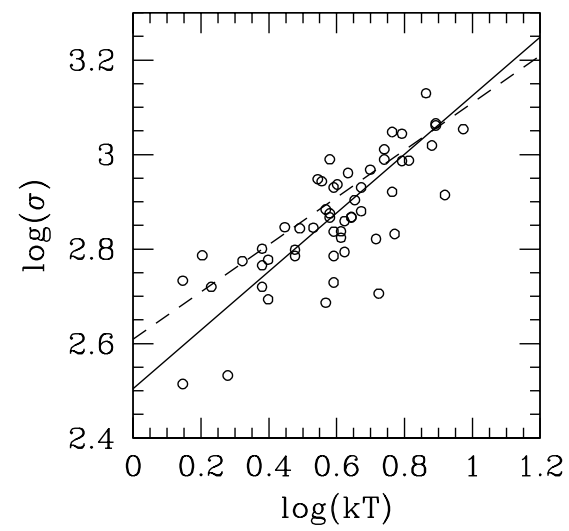

Fig. 6. - The solid line is the fit (weighted regression line) on 55 clusters. The dashed line represents the model with $\beta_{\text {spec }}=1$.

Although our work is not devoted to the analysis of galaxy distribution, we have had to address this issue in the computation of cluster masses. By using a King-like profile we obtain good alternative estimates of individual virial radii (see Figure 1, top-left panel), which is our aim in order to obtain reliable cluster mass estimates. Moreover, when one or two parameters $\left(\alpha, R_{c}\right)$ are fixed, the estimate of virial radii result less good, supporting the existence of an intrinsic spread of cluster parameters.

As for the comparison with previous King-like fits, we find good agreement with the value of the exponent $\alpha$ obtained by $\mathrm{G} 95\left(\alpha=0.8_{-0.1}^{+0.3}\right)$ and with other previous estimates (see Bahcall \& Lubin 1994 and references therein). Instead, we find typically smaller core radii $R_{c}$ (cf. our median $R_{c}=0.05 h^{-1} M p c$ with $R_{c}=0.17 h^{-1} M p c$ of G95). We verify that this difference is due to the fact that we determine cluster centers from the galaxy density peak (by using the two-dimensional kernel adaptive method, e.g. Girardi et al. 1996; Pisani 1996) rather than from averaging galaxy coordinates. Our finding is in agreement with the suggestion of Beers \& Tonry (1986) that large core radii can be produced by inaccurate cluster centers. Noticeably our small values for $R_{C}$ are in agreement with core radii relative to the total mass distribution derived from gravitational lensing (upper limits from 30 to $65 \mathrm{~h}^{-1} \mathrm{kpc}$, Narayan \& Bartelmann 1997).

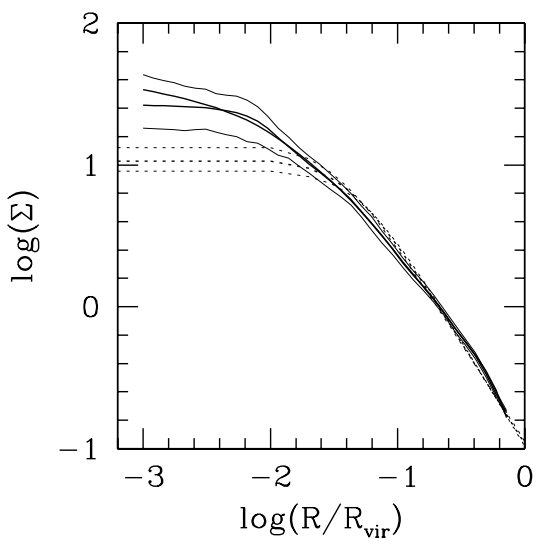

Fig. 7. - The comparison of the fits to the (normalized) combined projected galaxy number density $\Sigma$. The dotted line gives the result of the King-like profile fit with its error bands (faint dotted lines). The solid lines give the results of the nonparametric (MPL) fit with two smoothing parameters; the more smoothed solution showing a stronger gradient (in central regions) than the less smoothed solution for which we also give the bootstrap error bands (at the $95 \%$ s.l., faint solid lines).

However, the question of the existence of a core radius in the galaxy distribution is still open. For instance, Merritt \& Tremblay (1994) find no evidence for a core in Coma clusters (by using about 1500 galaxies), whilst Adami et al. (1998c) found that King profile better fits the ENACS clusters than do de Vaucouleurs or Navarro et al. (1996) profiles (see also Adami et al. 1998b). Indeed, we use the King-like profile in order to obtain information for individual clusters, but, to go deeper in the "core radius" issue we use a nonparametric method to fit the galaxy number density obtained by combining together the galaxies of all well sampled clusters (92 clusters sampled up to $R_{v i r}$, see $\oint 4.3$ ) and normalizing distances to $R_{v i r}$. In particular, we use the Maximum Penalized Likelihood (MPL) method (Merritt \& Tremblay 1994) which gives accurate representations of the true density profile according to 
the authors. Figure 7 compares the fits to the surface galaxy number density: the result of the King-like profile $\left(R_{c}=(0.04 \pm 0.01) \times R_{v i r}, \alpha=0.72 \pm 0.03\right)$, and the MPL fits. For MPL we show two estimates derived using two different smoothing parameters $\left(\lambda=10^{-4}, 10^{-5}\right.$; see Merritt \& Tremblay 1994 for details), which represent a compromise in computing a solution which is "most consistent" with the data, but not unacceptably noisy; the real features are those that persist over a wide range of choices for the smoothing. The existence of a core radius, being dependent on the choice of the smoothing parameter, is questionable. There is no evidence for a core radius as large as the one fitted by the King-like profile, but rather there is an indication for a smaller core radius $\left(\sim 0.01 \times R_{v i r} \sim 10\right.$ $\left.h^{-1} k p c\right)$ or, alternatively, no core radius. It is difficult to address this issue since we are dealing with dimensions close to galaxy sizes. However, the outer $\left(R \gtrsim 50 h^{-1} k p c\right)$ profile given by both the MPL estimates is in acceptable agreement with the profile of the King-like fit corroborating our use of the King-like profiles in estimating "global" quantities.

\section{SUMMARY AND CONCLUSIONS}

The main points of this work may be summarized as follows:

i) We evaluate in a homogeneous way the optical masses of 170 nearby clusters $(z \leq 0.15)$. This sample, which is the largest set of clusters up to now analyzed in the literature, includes both data from the literature and the new ENACS data (Katgert et al. 1996, 1998).

ii) On the assumption that mass follows the galaxy distribution, we compute the masses of each cluster by applying the virial theorem to the member galaxies and we verify our results by using the Jeans equation.

iii) Our mass estimates are smaller than previous optical estimates. This fact is due both to our better membership assignment procedure and to the application of the correction due to the presence of the surface term in the virial theorem (recently stressed by Carlberg et al. 1997a). iv) After appropriate rescaling to the X-ray radii, we compare our optical mass estimates to those derived from X-ray analyses, which we have compiled from the literature (for 66 clusters). We find a good overall agreement.

v) The above agreement is expected on the basis of two common assumptions: a) that mass follows the galaxy distribution, b) that clusters are not far from a situation of dynamical equilibrium with both gas and galaxies reflecting the same underlying mass distribution. It should be pointed out that Carlberg et al. (1997a) have recently drawn similar conclusions for a sample of distant clusters (the CNOC sample). In particular, we find evidence for a galaxy distribution which is colder and less extended than the gas distribution.

Several recent studies have casted doubts on cluster mass estimates and attempted to lower the cluster baryon fraction by reducing the cluster masses (e.g., Gunn \& Thomas 1996; Wu \& Fang 1996). We stress that our study strongly supports the reliability of present cluster mass estimates derived from X-ray analyses and/or (appropriate) optical analyses. Hence, it is even more difficult to reconcile present data with a $\Omega_{0}=1$ Universe (e.g. White et al. 1993b).

Our cluster masses are suitable for statistical studies. In particular, we did not reject a priori those clusters with a poor number of selected members, which usually have a small mass, in order to avoid having a final cluster sample biased towards more massive systems.

We are particularly indebted to the ENACS team for having kindly provided us with their data, based on observations collected at the European Southern Observatory, in advance of publication.

We thank Dario Fadda for useful discussions and the anonymous referee for useful suggestions. This work has been partially supported by the Italian Ministry of University, Scientific Technological Research (MURST), by the Italian Space Agency (ASI), and by the Italian Research Council (CNR-GNA).

\section{REFERENCES}

Abell, G.O., Corwin, H. G. Jr., \& Olowin, R. P. 1989, ApJS, 70,1

Adami, C., Biviano, A., \& Mazure, A. 1998a, A\&A, 331, 439

Adami, C., Mazure, A., Biviano, A., \& Katgert, P. 1998b, A\&A, submitted

Adami, C., Mazure, A., Biviano, A., Katgert, P., \& Rhee, G. 1998c, A\&A, 331,493

Allen, S. W. 1997, preprint astro-ph/9710217

Allen, S. W. Fabian, A. C., \& Kneib, J. P. 1996, MNRAS279, 615

Bahcall, N. A., \& Cen, R. 1993, ApJ, 407, L49

Bahcall, N. A., \& Lubin, L. M. 1994, ApJ, 426, 513

Bardelli, S., Zucca, E., Malizia, A., Zamorani, G., Scaramella, R., \& Vettolani, G. 1996, A\&A, 305, 435

Bardelli, S., Zucca, E.,Vettolani, G., Zamorani, G., Scaramella, R., Collins, C. A., \& MacGillivray, H. T. 1194, MNRAS, 267, 665

Bartelmann, M., \& Steinmetz 1996, MNRAS, 283, 431

Bartelmann, M., Steinmetz, \& Weiss 1995, A\&A, 297, 1

Beers, T. C., Flynn, K., \& Gebhardt, K. 1990, AJ, 100, 32

Beers, T. C., Forman, W., Huchra, J. P., Jones, C., \& Geb- hardt, K. 1991, AJ, 102, 1581

Beers, T. C., Gebhardt, K., Huchra, J.P., Forman, W., Jones, C. \& Bothun, G. D. 1992, ApJ, 400, 410

Beers, T. C., Geller, M. J. , Huchra, J. P., Latham, D. W., \& Davis, R.J. 1984, ApJ, 283, 33

Beers, T. C., \&, Tonry, J. L. K. 1986, AJ, 300, 557

Binggeli, B., Sandage, A., \& Tammann, G. A. 1985, AJ, 90, 1681

Binney, J., \& Tremaine, S. 1987, in "Galactic Dynamics", ed. J. P. Ostriker, Princeton University Press, New Jersey

Bird, C. M. 1995, ApJ, 445, L81, (B95)

Bird, C. M., Mushotzky, R. F, \& Metzler, C. A. 1995, ApJ, 453,40

Biviano, A., Girardi, M., Giuricin, G., Mardirossian, F., \& Mezzetti, M. 1992, ApJ, 396, 35

Biviano, A., Girardi, M., Giuricin, G., Mardirossian, F., \& Mezzetti, M. 1993, ApJ, 411, L13

Borgani, S. et al. 1997, New Astronomy, 1, 321

Bothun, D., Aaronson, M., Schommer, B., Mould, J., Huchra, J., \& Sullivan, W. T. III 1985, ApJS, 57, 423 
Bothun, G. D., \& Schombert, J. M. 1988, ApJ, 335, 617

Briel, U. G., \& Henry, J. P. 1994, Nature, 372, 439

Briel, U. G., \& Henry, J. P. 1996, ApJ, 472, 131

Briel, U. G., Henry, J. P., \& Boehringer, H. 1992, A\&A, 259, L31

Buote, D. A., \& Canizares, C. R. 1996, ApJ, 457, 565

Butcher, H. R., \& Oemler, A. Jr. 1985, ApJS, 57, 665

Carlberg, R. G., Yee, H. K. C., \& Ellingson, E. 1997a, ApJ, 478,462

Carlberg, R. G., Yee, H. K. C., Ellingson, E., Abraham, R., Gravel, P., Morris, S., \& Pritchet, C. J., et al. 1996, ApJ, 462,32

Carlberg, R. G., Yee, H. K. C., Ellingson, E., Morris, S. L., Abraham, R., Gravel, P., Pritchet, C. J., et al. 1997b, ApJ, 476, L7

Cavaliere, A., \& Fusco-Femiano, R. 1976, A\&A, 49, 137

Cavaliere, A., Menci, N. \& Tozzi, P. 1997, ApJ, 484, L21

Chapman, G. N. F., Geller, M.J., \& Huchra, J. P. 1987, AJ, 94,571

Chapman, G. N. F., Geller, M.J., \& Huchra, J. P. 1988, AJ, 95, 999

Chincarini, G., \& Rood, H. J. 1976, PASP, 88, 388

Chincarini, G., Tarenghi, M., \& Bettis, C. 1981, A\&A, 96, 106

Cirimele, G., Nesci, R., \& Trevese, D. 1997, ApJ, 475, 11

Colless, M., \& Hewett, P. 1987, MNRAS, 224, 453

Collins, C. A., Guzzo, L., Nichol, R. C., \& Lumsden, S. L. 1995, MNRAS, 274, 1071

Cowie, L. L., Henriksen, M., \& Mushotzky, R. 1987, ApJ, 317, 593

Cristiani, S., de Souza, R., D’Odorico, S., Lund, G., \& Quintana, H. 1987, A\&A, 179, 108

Cruddace, R. G., Kowalski, M. P., Fritz, G. G., Snyder, W. A., Fenimore, E. E., \& Ulmer, M. P. 1997, ApJ, 476, 479

Danese, C., De Zotti, G., \& di Tullio, G. 1980, A\&A, 82, 322

David, L. P., Jones, C., \& Forman, W. 1995, ApJ, 445, 578

David, L. P., Slyz, A., Jones, C., \& Forman, W., Vrtilek, S. D., \& Arnaud, K. A. 1993, ApJ, 412, 479

Dell'Antonio, I. P., Geller, M. J., \& Fabricant, D. G. 1994, AJ, 107,427

Dell'Antonio, I. P., Geller, M. J., \& Fabricant, D. G. 1995, AJ, 110,502

den Hartog, R., \& Katgert, P. 1996, MNRAS, 279, 349

Dejonghe, H. 1987, MNRAS, 224, 13

Dickens, R. J., Currie, M. J.,\& Lucey, J. R. 1986, MNRAS, 220, 679

Dickens, R. J., \& Moss, C. 1976, MNRAS, 174, 47

Dressler, A., \& Shectman, S.A. 1988a, AJ, 95, 284

Dressler, A., \& Shectman, S. A. 1988b, AJ, 95, 985

Durret, F., Gerbal, D., Lachiéze-Rey, M., Lima-Neto, G., \& R. Sadat 1994, A\&A, 287, 733

Escalera, E., Biviano, A., Girardi, M., Giuricin, G., Mardirossian, F., Mazure, A., \& Mezzetti, M. 1994, AJ, 423,539

Ettori, S., Guzzo, L., Tarenghi, M. 1995, MNRAS, 276, 689

Evrard, A. E., Metzler, C. A., \& Navarro, J. F. 1996, ApJ, 469, 494

Eyles, C. J., Watt, M. P., Bertram, D., Church, M. J., Ponman, T. J., Skinner, G. K., \& Willmore, A. P. 1991, ApJ, 376, 23

Faber, S. M., \& Dressler, A. 1977, AJ, 82, 187

Fabricant, D. G., Kent, S. M., \& Kurtz, M. J. 1989, ApJ, 336,

Fabricant, D., Kurtz, M., Geller, M., Zabludoff, A., Mack, P., \& Wegner, G. 1993, AJ, 105, 788

Fadda, D., Girardi, M., Giuricin, G., Mardirossian, F., \& Mezzetti, M., A., 1996, ApJ, 473670 (F96)

Fort, B. 1994, A\&A Rev., 5, 239

Frenk, C. S., Evrard, A. E., White, S. D. M., \& Summers, F J. 1996 , Aिग, 472,460

Gavazzi, G. 1987, ApJ, 320, 96

Geller, M. J., Beers, T. C., Bothun, G. D., \& Huchra, J. P. 1984, AJ, 89, 319

Gerbal, D., Durret, F., Lima-Neto, G., \& Lachiéze-Rey, M. 1992, A\&A, 253, 77

Gerbal, D., Durret, F., \& Lachiéze-Rey, M. 1994, A\&A, 288, 746

Giovanelli, R., Haynes, M. P., \& Chincarini, G. L. 1982, ApJ, 262,442

Girardi, M., Borgani, S., Giuricin, G., Mardirossian, F., \&
Mezzetti, M., 1998, submitted to ApJ

Girardi, M., Escalera, E., Fadda, D. Giuricin, G., Mardirossian, F., \& Mezzetti, M., 1997a, ApJ, 482, 41

Girardi, M., Fadda, D., Escalera, E., Giuricin, G., Mardirossian, F., \& Mezzetti, M., 1997b, ApJ, 490, 56

Girardi, M., Fadda, D., Giuricin, G., Mardirossian, F., Mezzetti, M., \& Biviano, A. 1996, ApJ, 457, 61

Girardi, M., Biviano, A., Giuricin, G., Mardirossian, Mezzetti, M., A. 1995, ApJ, 438, 527 (G95)

Giuricin, G., Mardirossian, F., \& Mezzetti, M. 1982, ApJ, 259, 30

Gregory, S. A., \& Thompson, L. A. 1984, ApJ, 286, 422

Gregory, S. A., Thompson, L. A., \& Tifft, W. G. 1981, ApJ, 243,411

Gross, M. A. K., Somerville, R. S., Primack, J. R., Borgani, S., Girardi, M. 1998, in preparation

Grossman, S. A., \& Narayan, R. 1989, ApJ, 344, 637

Gunn, J. E., \& Gott, J. R. III 1972, ApJ, 176, 1

Gunn, K. F., \& Thomas, P. A. 1996, MNRAS, 281, 1133

Heisler, J., Tremaine, S., \& Bahcall, J. N. 1985, ApJ, 298, 8

Hill, J. M.,\& Oegerle, W. R. 1993, AJ, 106, 831

Hintzen, P. 1980, AJ, 85, 626

Hintzen, P., Hill, J. M., Lindley, D., Scott, J. S., \& Angel, J. R. P. 1982, AJ, 87, 1656

Hintzen, P., \& Scott, J.S. 1979, A\&A, 74, 116

Huchra, J., Geller, M., Clemens, C., Tokarz, S., \& Mickel, A. 1992, Bull. CDS, 41, 31

Huang, Z. \& Sarazin, C. L. 1996, ApJ, 461, 622

Hughes, J. P. 1989, ApJ, 337, 21

Katgert, P., Mazure, A., den Hartog, R., Adami, C., Biviano, A., \& Perea, J., 1998, A\&AS, 129, 399

Katgert, P., et al. 1996, A\&A, 310, 8

Kent, S. M., \& Gunn, J. E. 1982, AJ, 87, 945

Kent, S. M., \& Sargent, W. L. W. 1983, AJ, 88, 697

Knopp, G. P., Henry, J. P., \& Briel, U. G. 1996, ApJ, 472, 125

Ikebe, Y. et al. 1996, Nature, 379, 427

Jones, C., \& Forman, W. 1984, ApJ, 276, 38

Lauberts, A., Valentjin, E.A. 1989, "The Surface Photometry Catalogue of the ESO-Uppsala Galaxies", Garching bei Munchen: ESO

Liang, H., Pierre, M., Unewisse, A., \& Hunstead, R. W. 1997, A\&A321, 64

Limber, D. N., \& Mathews, W. G. 1960, ApJ, 132, 286

Loeb, M., \& Mao, S. 1994, ApJ, 435, L109

Loewenstein, M., \& Mushotzky, R. F. 1996, ApJ, 471, L83

Lucey, J. R., \& Carter, D. 1988, MNRAS, 235, 1177

Lucey, J. R., Dickens, R. J., Mitchell, R. J., \& Dawe, J. A. 1983, MNRAS, 203, 545

Malumuth, E. M., Kriss, G. A., Van Dyke Dixon, W., Ferguson, H. C., \& Ritchie, C. 1992, AJ, 104, 495

Markevitch, M., \& Vikhlinin, A. 1997, ApJ, 491, 467

Materne, J., \& Hopp, U. 1983, A\&A, 124, L13

Mazure, A., et al. 1996, A\&A, 310, 31

McGlynn, T. A., \& Fabian, A. C. 1984, MNRAS, 208, 709

Merritt, D. 1987, ApJ, 313, 121.

Merritt, D. 1988, in The Minnesota Lectures on Clusters of Galaxies and Large-Scale Structures, ed. J. M. Dickey (ASP Conf. Ser., 5), p. 175

Merritt, D. Gebhardt, K. 1994, in "Clusters of Galaxies", eds. F. Durret, A. Mazure, and J. Tran Than Van, (ed. Frontieres), Gif-sur-Yvette Cedex - France, p. 11

Merritt, D. Tremblay, B. 1994, AJ, 108, 514

Metcalfe, N., Godwin, J. G., \& Spenser, S. D. 1987, MNRAS, 225,581

Metzler, C. A., \& Evrard, A. E. 1997, preprint astro$\mathrm{ph} / 9710324$

Mezzetti, M. et al. 1998, in preparation

Miralda-Escudé, J. 1993, ApJ, 403, 497

Miralda-Escudé, J., \& Babul, A. 1995, ApJ, 449, 18

Miyaji, T. et al. 1993, ApJ, 419, 66

Mushotzky, R. F., Loewenstein, M., Arnaud, K., \& Fukazawa Y. 1995, in Dark Matter, ed. S. S. Holt \& C. L. Bennett (New York: AIP), p. 231

Narayan, R., \& Bartelmann, M. 1997 to appear in Formation of Structure in the Universe, Proceedings of the 1995 Jerusalem Winter School; edited by A. Dekel and J.P. Ostriker; Cam- 
bridge University Press; preprint astro-ph/9606001

Navarro, J. F., \& Frenk, C. S., White, S. D. M., ApJ, 462, 563

Nulsen, P. E. J., \& Boehringer, H. 1995, MNRAS, 274, 1093

Oegerle, W. R., \& Hill, J. M. 1993, AJ, 104, 2078

Oegerle, W. R., Hill, J. M., \& Fitchett, M. J. 1995, AJ, 110, 1

Ostriker, E. C., Huchra, J. P., Geller, M. J., \& Kurtz, M. J. 1988, AJ, 96, 1775

Perea, J., del Olmo, A., \& Moles, M. 1990, A\&A, 237, 319

Pinkney, J., Rhee, G., \& Burns, J. O. 1993, ApJ, 416, 36

Pinkney, J., Roettiger, K., Burns, J. O., \& Bird, C. A. 1996, ApJS, 104, 1

Pisani, A. 1993, MNRAS, 265, 706

Pisani, A. 1996, MNRAS, 278, 697

Pislar V., Durret F., Gerbal D., Lima Neto G.B., \& Slezak E. 1997, A\&A, 322, 53

Plionis, M., Barrow, J. D., Frenk, C. S. 1991, MNRAS, 249, 662

Postman, M., Huchra, J. P., M., \& Geller, M. J. 1986, AJ, 92, 1238

Press, W. H., Teukolsky, S. A., Vetterling, W. T., \& Flannery, B. P. 1992, Numerical Recipes (2d ed.; Cambridge: Cambridge Univ. Press)

Proust, D., Quintana, H., Mazure, A., da Souza, R., Escalera, E., Sodré, L. Jr., \& Capelato, H. V. 1992, A\&A, 258, 243

Quintana, H., \& de Souza, R. 1993, A\&AS, 101, 475

Quintana, H., Melnick, J., Infante, L., \& Thomas, B. 1985, AJ, 90,410

Quintana, H., \& Ramirez, A. 1990, AJ, 100, 1424

Richter, O. G. 1987a, A\&AS, 67, 237

Richter, O. G. 1987b, A\&AS, 261, 266

Richter, O. G. 1989, A\&AS, 77, 237

Richter, O.G., \& Huchtmeier, W. K. 1982, A\&A, 109, 155

Roettiger, K., Burns, J., \& Locken, C. 1993, ApJ, 413, 492

Roettiger, K., Stone, J. M., \& Mushotzky, R. F. 1998, ApJ, 493, 62

Schindler, S. 1996a, A\&A, 305, 756

Schindler, S. 1996b, MNRAS, 280, 309

Schindler, S., \& Almudena Prieto, M. 1997, A\&A, 327, 37

Scodeggio, M., Solanes, J. M., Giovanelli, R., \& Haynes, M. P.
1995, ApJ, 444, 41

Sharples, R. M., Ellis, R. S., \& Gray, P. M. 1988, MNRAS, 231,479

Sodré, L., Capelato, H. V., Steiner, J. E., Proust, D., \& Mazure, A. 1992, MNRAS, 259, 233

Squires, G., \& Kaiser, N. 1996, ApJ, 473, 65

Stepanyan, Dzh. A. 1984, Astrophysics, 20, 478

Stein, P. 1997, A\&A, 317, 670

Teague, P. F., Carter, D., \& Gray, P. M. 1990, ApJS, 72, 715

The, L. S., \& White, S. D. M. 1986, AJ, 92, 1248

Tully, R.B. 1988, "Nearby Galaxy Catalog" (Cambridge: CUP)

Watt, M. P., Ponman, T. J., Bertram, D., Eyles, C. J., Skinner, G. K., \& Willmore, A. P. 1992, MNRAS, 258, 738

West, M. J. 1994, in Clusters of Galaxies, eds. F.Durret, A.Mazure, and J.Tran Thanh Van, p.23

White, D. A., \& Fabian, A. C. 1995, MNRAS, 273, 72

White, R. E. III 1991, ApJ, 367, 69

White, D. A., Jones, C., \& Forman, W. 1997, MNRAS, 292 , 419

White, S. D. M., Efstathiou, G., \& Frenk, C. S., 1993a, MNRAS, 262, 1023

White, S. D. M., \& Frenk, C. S., 1991, ApJ, 379, 52

White, S. D. M., Navarro, J. F., Evrard, A. E., \& Frenk, C. S., 1993b, Nature, 366, 429

Willmer C. N. A., Focardi P., Chan R., Pellegrini P. S., \& Nicolaci Da Costa, L. 1991, AJ, 101, 57

Willmer C.N.A., Focardi P., Nicolaci Da Costa, L., Pellegrini, P. S. 1989, AJ, 98, 1531

Wu, X. P. 1994, ApJ, 436, L115

Wu, X. P., \& Fang, L. Z., 1996, ApJ, 467, L45

Wu, X. P., \& Fang, L. Z., 1997, ApJ, 483, 62

Zabludoff, A. I., Huchra, J. P., \& Geller, M. J. 1990, ApJS, 74,

Zabludoff, A. I., Geller, M. J., Huchra, J. P., \& Ramella, M. 1993a, AJ, 106, 1301

Zabludoff, A. I., Geller, M. J., Huchra, J. P., \& Vogeley, M. S. 1993b, AJ, 106, 1273

Zwicky, F. 1933, Helv. Phys. Acta, 6, 110 